Lessons from the Field

\title{
Analysis of Clinical Experience Using Kolb's Experiential Learning Theory: Postgraduate Medical Surgical Nursing Track Students in Selected Sub- Specialties at the University Teaching Hospital of Kigali, Rwanda
}

Vedaste Bagweneza $^{1 *}$, Collins Anita ${ }^{1}$, Isaac Nsanzamahoro ${ }^{1}$, Vestine Mukanoheli $^{1}$, Florian Bahaya ${ }^{1}$, Perpetue Niyitegeka ${ }^{1}$, Pauline Kabanyana ${ }^{1}$, Marie Josee Mwiseneza ${ }^{1}$, Tony Gaterega ${ }^{1}$, Agnes Kura ${ }^{1}$, Claude Twahirwa ${ }^{1}$, Marie Munezero ${ }^{1}$

${ }^{1}$ School of Nursing and Midwifery, College of Medicine and Health Sciences, University of Rwanda, Kigali, Rwanda

*Corresponding author: Vedaste Bagweneza. School of Nursing and Midwifery, College of Medicine and Health Sciences, University of Rwanda, Kigali, Rwanda. Email: vedavich1@yahoo.fr

\begin{abstract}
\section{Background}

Clinical experiences provide an opportunity for nursing students to integrate theoretical knowledge into practice, and analyze their learning using a theoretical model. Students do not take this opportunity unless it is intentionally included by the facilitators. These observations from the second cohort of medical surgical nursing students with their facilitators, regarding their clinical training period in selected sub-specialties at the University Teaching Hospital of Kigali (CHUK) aimed at developing students' analytical and observational skills while developing clinical skills.
\end{abstract}

\section{Methods}

The study involved nine medical surgical nursing students who were in different subspecialties at CHUK from $7^{\text {th }}$ January up to the $20^{\text {th }}$ February, 2019. Informed observational approach and concomitant reporting were used to validate their learning and gain clinical experience. The students used Kolb's Experiential Learning Theory to analyze their experience, with the focus on innovative skills.

\section{Results}

All medical surgical nursing students reported that they improved their knowledge and skills during clinical practice, while broadening their outlook.

\section{Conclusion}

Kolb's Experiential Learning Theory is useful in validating and bridging theoretical learning to clinical practice.

Rwanda J Med Health Sci 2021;4(3):430-436

Keywords: Clinical experience, Experiential Learning, Kolb's Experiential Learning Theory

\section{Introduction}

The Human Resources for Health $(\mathrm{HRH})$ program contributes in the capacity building for both academic and clinical education programs in Rwanda, especially in nursing and midwifery domains.[1] Eight Masters of Science in Nursing tracks commenced in August 2015 in collaboration with the Ministry of Health (MOH) in the University of Rwanda, and Medical Surgical Nursing Track is one of them. The current study was conducted by the students and facilitators of the Medical Surgical Track.
Medical Surgical track is a clinical specialty, which means that they conduct field activities known as clinical practice.

Clinical experience has various forms and is the beginning of clinical learning process that broadens throughout the nursing student education.[2] Medical-surgical nursing constitutes the single largest nursing specialty in the United States, and in almost all countries, including Rwanda [3] because the registered nurses in this specialty manage adult patients with a wide variety of medical and surgical problems.[3] 
Focused clinical II in CHUK intended to help Medical Surgical students em-ploy an expanded use of the nursing process in the care of patients in ortho-pedic surgery, emergency department, burns unit, renal unit, operating the-atre, recovery and neurosurgery, with inclusiveness of patients' families in the plan of care. During this clinical practice, the medical surgical students and their facilitators had time to concurrently observe both the intervention they provided and its outcome on the hospitalized patients, and from that experience, they acquired different skills and clinical experience.

Fundamentally, this is one way for postgraduate nursing students to get the best real-world experiences and put into practice theoretical knowledge learnt in class. At this moment, student nurses start to develop higher competencies, enhance clinical thinking skills and receive specialty expertise in different hospital departments, and supportive clinical environment is re-quired for the students to gain enough nursing knowledge and skills. [4]

Medical-surgical nurses work with patients in various clinical settings, have impressive skills with vast knowledge of disease states and body sys-tems.[3] They are skilled and knowledgeable about many aspects of human health, allowing them to advocate for patients if needed. Medical surgical nurses may practice in almost all the services and departments of health fa-cilities, and therefore they need to pursue advanced degrees and get enough clinical experiences.

The master's program has been running from 2015. In addition, there are some graduates who completed Medical Surgical Nursing specialty. Despite that, the review of literature did not show any availability of clinical experiences reported by these medical surgical nurses, even if they all attended the clinical settings and managed patients in health facilities during their studies. Therefore, the aim of this article is to elaborate and highlight clinical experiences gained by medical surgical nursing students of the second cohort.

\section{Methods}

Postgraduate medical surgical nursing students used informed observational approach and concomitant reporting as they gained knowledge and clinical skills at CHUK from 7 th January up to 20th February, 2019. Prerequisites modules included Medical Surgical Nursing for Special Populations, Advanced Medical Surgical Nursing I, and Transforming Nursing Practice.
Along with the knowledge and skills gained from the module Focused Clinical I, it was possible to make clinical observations and provide nursing care for patients from the following subspecialties: Orthopedic, Neurosurgery, General Surgery, Emergency Wards and the Burns Unit and the Operating Theater at CHUK. The students were allocated in these subspecialties whereby each student had to spend at least 1 week in each subspecialty, and rotate to the next after a week. The students then chose patients of whom they wanted to observe and monitor the progress of the interventions. The main observation was to assess the progress of wounds that were treated by honey, and how patients reacted to the use of honey. The students also had an opportunity to observe, assess and provide nursing care to the clients with other medical and surgical conditions and interact with the nursing staff. The students did this referring to the learnt didactic modules about diseases, and medical and nursing care in that semester.

Observational research is a type of nonexperimental studies in which behavior is systematically observed and recorded. Its goal is to describe a variable or set of variables, in order to obtain a clear view of specific characteristics of an individual, group, or setting. In such a study nothing is manipulated or controlled, and as such one cannot arrive at causal conclusions using this approach. The data that are collected in observational research studies are often qualitative in nature but they may also be quantitative or both (mixed-methods). There are several different types of observational research designs. One of them is naturalistic observation, which has also 2 types: disguised and undisguised (informed) naturalistic observation. In cases where it is not ethical or practical to conduct disguised naturalistic observation, researchers conduct undisguised naturalistic observation where the participants are made aware of the researcher's presence and the monitoring activity of their behavior. In this study, the students and facilitators made the patients or nurses part of their learning experience, to be observed.

The students used Kolb's Experiential Learning Theory to analyze their experiences, which explains experiential learning as a cyclical process that begins anywhere in the cycle and follows the sequence of knowledge acquisition based on experiences. Effective learning takes place when students pass through each of the four stages of the cycle several times.[5] This theory was chosen because it helps the students to integrate the experiential leaning skills 
into concise and logical approach, provides the chance for the students to practice the skills and develop attitudes such as curiosity and self-direction.

The observations emphasized on the innovative skills during clinical prac-tice. These innovative skills consisted of use of honey and vinegar in wound management at CHUK, and the students assessed the relevance of these in-novative skills in wound management. They chose the hospitalized patients with wounds managed by honey and vinegar, and made a progressive obser-vation in the wound changes and healing process relative to the usual time the wound of the same type would take to heal. For that purpose, they measured the width or length of the wound during every wound care to track the wound healing progress. Finally, students had time to present their learning of new knowledge and skill acquisition to the facilitators, for discus-sion.

\section{Process of Learning as it relates to Kolb's Experiential Learning Theory and Relevant Clinical Observations}

David Kolb who developed the theory in 1970s and published his learning styles model in 1984,[6] is an American psychologist and educational theorist who earned his Bachelor degree of Arts from Knox College in 1961 and PhD in Psychology in 1976 from Harvard University. He received several research recognition awards and four honorary degrees recognizing his contributions to experiential learning in higher education, [7] including an award from the National Society of Experiential Education in 2008. He founded the model based on four distinct elements which are concrete experience, observation of new experience, abstract conceptualization and active experimentation, [7] which were used by medical surgical nursing students.

\section{First stage of Kolb's experiential learning theory: Concrete Experience}

With this stage, a new experience or situation is encountered, on a reinterpretation of existing experience.[6] While in burns unit and surgical ward, students encountered a new situation: treating wound using honey. This was not common for the students who were already nurses working in different institutions, but were not aware that using honey in wound management would yield a tangible result. The students were in this stage when they noticed honey was being used to treat patients' wounds. [8]
Literature shows that honey has broad-spectrum anti-infectious activities against at least 80 species of micro-organisms including gram positive and gram-negative bacteria, aerobes and anaerobes. The use of this product of bees shortens the period of wound healing and its anti-infectious property for the treatment of infected wounds, burns and other skin conditions was confirmed in other studies. [911] In addition, it was proven that honey has antiinflammatory and antioxidant properties, boosting effect on immune system, debridement action and stimulating role in wound regeneration.[12] They reasoned that honey reduces inflammation and in turn, should reduce pain sensation.

The honey used in CHUK was bought from shops and stored by the patients themselves. The sterility of this honey and quantity used are questionable because, the way this product is conserved and used is not standardized, and likelihood of microbial contamination cannot be ruled out. This is supported by the findings of the study conducted in Pakistan in 2015 which highlighted that honey can get contaminated if standard sanitation and manufacturing process are not well controlled.[13] They found that the source of infection included human, dirty equipment, honey container, wind and dust. However, the study showed that if stored properly, growth of microorganism in honey was difficult, especially due to the high osmolarity, acidity and the presence of hydrogen peroxide in natural honey.[13]

In literature, the students discovered the most common type of honey used in wound dressing was extracted from the Manuka tree (Leptospermum scoparium) due to its non-peroxide antimicrobial and anti-inflammatory effects as well as ability to shorten the length of wound healing. $[8,14,15]$ The students discovered vinegar was being used when Pseudomonas aeruginosa bacteria was grown in the culture from the wound's exudates. Unlike use of honey, the awareness of vinegar use in wound healing in Rwanda is limited. CHUK is one of the national referral hospitals of Rwanda that adopted it as a wound cleansing agent. Research showed that vinegar which has been used since many centuries as an important cooking constituent, is used in persistent nonhealing (chronic) wounds resistant to any sort of treatment. The most common pathogen responsible for chronic wound, Pseudomonas aeruginosa which is an opportunistic bacterium found on the skin but resistant to many antimicrobials, is also susceptible to vinegar.[16] This finding supports the practice in CHUK, because they used vinegar in management of chronic wounds, and this can then be considered as evidence based practice. 
The students discovered vinegar was being used when Pseudomonas aeruginosa bacteria was grown in the culture from the wound's exudates. Unlike use of honey, the awareness of vinegar use in wound healing in Rwanda is limited. CHUK is one of the national referral hospitals of Rwanda that adopted it as a wound cleansing agent. Research showed that vinegar which has been used since many centuries as an important cooking constituent, is used in persistent non-healing (chronic) wounds resistant to any sort of treatment. The most common pathogen responsible for chronic wound, Pseudomonas aeruginosa which is an opportunistic bacterium found on the skin but resistant to many antimicrobials, is also susceptible to vinegar. [16] This finding supports the practice in CHUK, because they used vinegar in management of chronic wounds, and this can then be considered as evidence based practice.

In emergency department, students made a startling discovery. They found that manyinjuries in summer among children were secondary to falling down from the height of the fruit trees as a result of many them playing unsupervised more often. The healthcare providers at CHUK call it the "avocado period". The students were saddened to see that some of these children sustained traumatic brain injuries (TBI) due to falling down from avocado and guava trees and developed complications like brain herniation, hemorrhage and some even died. On learning this, the students investigated further and found that the phenomenon was common in other parts of the world as well. For example, in Nepal a study conducted in 2015 titled "Epidemiology of Unintentional Child Injuries in the Makwanpur District of Nepal: A Household Survey" revealed that, falls are the leading cause of non-fatal injury for all age groups, most often from trees, balconies, ladders or edges of fields. [17] Similarly, literature specifies that the falls are the major causes of injuries among children, as they can be endangered while exploring the environment and climbing heights.[18] The nursing students realized that there was an opportunity to address this issue with parents and children, as the problem could be prevented by educating them. As a group, they wondered whether this issue needed to be addressed to other stakeholders in the wider community such as parents, teachers and children as well. In the orthopedic ward, from 1st to 30th January 2019 (30 days), the total number of patients admitted for orthopedic surgery was 45, of whom 30 were hospitalized due to road traffic accidents (RTA), especially from motor-vehicle accident (MVA).
Currently, RTAs are the 8th leading cause of death worldwide for all age groups, with most deaths occurring in Africa where Rwanda is located,[19, 20] and in the age range of 20 to 35 years. In modeled estimates, Rwanda was found to be having the highest road traffic fatality rate at 22 deaths per 100,000 with the highest burden of road traffic injuries standing at 1,173 disabilityadjusted life years per 100,000 population compared to other East African countries.[21] Data from 2015 show that 2,508 people were injured with 366 recorded deaths.[20] Many students observed that these patients developed complications such as deep vein thrombosis (DVT), malunion of the bones, chronic pain, infection and constipation.

In burns unit, the majority of patients admitted were children and for most of them, the cause of burn was accident related to spilling of hot water, hot tea and porridge. After gathering all information through history taking, the students found that many children were left at home by their parents, being cared for by other children who did not even know how to protect themselves or their siblings from burns. The students provided education to patients' next of kin regarding children safety at home. In this phase of learning, the students and facilitators used education as a tool to improve care and prevent injuries.

\section{Second stage of Kolb's experiential learning theory: Observation of the new experience}

In this stage, the learners reflect on the experience prior to making any judgment.[6] Students reflected on the various experiences from rotation in different services at CHUK, including making reference to the clinical prerequisite modules, and all the materials that facilitated the learning process in the module Focused Clinical II.

Moreover, students got the point that honey could be used in wound management. However, with reference to the previous experience in honey use, students then reflected on patients who were complaining of pain during the application of honey to their wounds and they noticed that a patient in burns unit who was on ketamine did not complain of pain during removal of dressing and cleaning of the wound, even during the application of the honey. Therefore, the students delved into their learning experience from the earlier modules they had undertaken and advocated for the use of non-pharmacological management of pain or the use of pain-relieving drugs before performing 
any painful procedure or applying honey in order to avoid unnecessary suffering to the patients. This observation provided the students with the stimulus to want to learn more about pain assessment and effective pain management before application of honey on wound.

In emergency department and burns unit, many surgical cases consisted of children under five years. The students learnt about predisposing factors, and they made a conclusion that these children were more prone to burns as a result of poor supervision from parents or guardians. The students then came up with the suggestion to undertake a project on community sensitization starting from the schools on the issue of less than 5 years old, the risk factors for, and prevention of burn injuries.

The students also encountered a patient with severe burn on the hand and forearm which he sustained while using hot press for clothes. The patient later developed compartment syndrome which was treated with fasciotomy. They newly learned that the compartment syndrome is a serious surgical emergency complication of extremity circumferential burns, that results from the irregularities in the functioning and circulation of the tissue because of the increased interstitial tissue pressure in a limited fascial area. [22, 23] Students realized that, in orthopedic ward, male admissions were more compared to women. Most of them being young men who drove motorcycles. It came to their mind that road traffic accidents (RTAs) could be mitigated only if a multidisciplinary team approach is undertaken involving the transport federation and cooperatives, the police, the community, and the community health nurses and workers, who have access to young Rwandan men.

\section{Third stage of Kolb's experiential learning theory: Abstract conceptualization}

This stage highlights how learners can reflect and provide a new idea or make modification of an existing abstract concept,[6] Clinical practice was the platform for the students to use abstraction from concrete experience gained during clinical practice in CHUK and it served them to come up with new ideas. Through this learning process, the students realized that, application of honey led to pain even though literature highlighted that the use of honey reduces pain. Patients suffering from pain cannot be ignored, as several students observed at different times with different patients that the pain experience was specific to application of honey and not to cleansing with saline and dressing. The students also observed that patients suffered a lesser degree of pain on subsequent days of use of honey during dressing. Furthermore, the students also found through their day to day observations that there was good improvement in wound healing compared to other methods of wound dressing. The students ascertained that both infected and deep clean wounds were selected for the use of honey innovative technique at CHUK. This is supported by the literature which showed that a wide range of types of wounds were selected for use of honey during dressing.[15]

The students abstracted that this would be a good area for research for the medical surgical nursing track students. After exhausting all other non-pharmacological treatments for pain, as a last resort, the students contemplated whether analgesics such as non-steroidal antiinflammatory drugs (NSAIDS) and opioids could be used prior to honey application to ease pain experienced during wound dressing. The students also abstracted that they would suggest to the staff nurses to adhere to the universal precautions of honey storage in order to prevent the introduction of microorganisms in honey or prevent the honey from deterioration. This is because it has been identified that the quality of honey can be altered if stored in inappropriate conditions or if microorganisms gain entry into the honey.[24,25] It was found that the quality of honey reduced if it was stored in extreme conditions, either too hot or too cold temperatures; and a study on "the effect of storage temperature on the quality of honey" revealed that the ideal temperatures for honey storage is room temperature (260 centigrade). Fifty degrees centigrade and \pm 5 degrees centigrade were not suitable for its storage.[24]

Literature revealed that vinegar use contributed a great deal in the healing of the wound which was found to be colonized by pseudomonas aeruginosa, including chronic wounds such as diabetic wounds, with the recommended concentration being $1 \% .[16,26]$ They realized that it would be beneficial to establish protocol for both honey and vinegar use that clearly instruct the amount to be used and expected duration so that all healthcare providers can use them accordingly. 


\section{Fourth stage of Kolb's experiential learning theory: Active experimentation}

This stage highlights how the learners apply acquired experience to the world around them to see what can be the results. [6] As all the 9 students were together in the same hospital during the module Focused Clinical II, it facilitated them to gain more knowledge because each student made his/her observation in different services, using a reflective journal. The reflective journal is a compiled document that a student fills on a daily basis, and the students meet their clinical instructors every week to show the progress and discuss. This observational experience made in different hospital services aided the students to relate clinical experience with theory learned in class and post conference discussions with clinical instructors, where students and facilitators met after care delivery, trying to apply the learned knowledge into practice. They also planned to encourage use of honey and vinegar in other health facilities as they found these products to be good for wound care. Finally, this learning experience fostered a sense of unity among the students as each student provided their personal experiences and supplemented them with review of literature on the use of honey and vinegar in wound management.

\section{Conclusion and Recommendations}

Throughout the clinical experience, knowledge and skills were acquired through observation and active learning. Nursing staff provided significant contribution, and the integration of theories into practice by the students was facilitated by the clinical instructors. Students had the opportunity to improve their critical thinking capacity in relation to medical and surgical conditions and associated complications resulting from inadequate knowledge, skills and lack of adequate clinical experience of the staff or the patients themselves.The students recommend extension of research-based evidence on different products used in wound management, particularly honey and vinegar. Finally, the students' clinical experience contributed to the education of the community to adhere to safety measures to protect children at home, and educate motocycle-taxi drivers to adhere to road traffic safety regulations

\section{Authors' contribution}

IN and VM contributed in conception and design of the work; revised the work critically; final approval of the version to be published, then TG, PK, JCT, PN, MCM, FB, MAK and MJM contributed in conception and design of the work; final approval of the version to be published.

\section{Conflicts of interest}

No conflicts of interest to declare in this manuscript.

This article is published open access under the Creative Commons Attribution-NonCommercial NoDerivatives (CC BYNC-ND4.0). People can copy and redistribute the article only for noncommercial purposes and as long as they give appropriate credit to the authors. They cannot distribute any modified material obtained by remixing, transforming or building upon this article. See https:// creativecommons.org/licenses/by-nc-nd/4.0/

\section{References}

1. Uwizeye G, Mukamana D, Relf M, et al. Building Nursing and Midwifery Capacity Through Rwanda's Human Resources for Health Program. Epub ahead of print 2017. DOI: $10.1177 / 1043659617705436$.

2. Baclig JT. Life Cycle of a Nurse: Clinical Nursing Experience, https://www.rn.com/ nursing-news/life-cycle-of-a-nurse-clinicalnursing-experience/ (2015).

3. Academy of Medical- Surgical Nurses. What is Medical-Surgical Nursing?, https://www. amsn.org/about-amsn/what-med-surgnursing (2021).

4. Kol E, Serpil İ. Determining the opinions of the first-year nursing students about clinical practice and clinical educators. Epub ahead of print 2018. DOI: 10.1016/j. nepr.2018.04.009.

5. Akella D. L earning together: Kolb' s experiential theory and its application. Epub ahead of print 2010. DOI: 10.5172/ jmo.16.1.100.

6. McLeod S. Kolb's Learning Styles and Experiential Learning Cycle, https://www. simplypsychology.org/learning-kolb.html (2017).

7. Kolb DA. Experiential Learning: Experience as the source of Learning and Development Second Edition, https://www.researchgate. net/publication/315793484_Experiential Learning_Experience_as_the_source_of Learning_and_Development_Second_Edition (2015).

8. Tsang AS, Dart AJ, Dart CM, et al. Mechanisms of action of manuka honey in an equine model of second intention wound healing: current thoughts and future directions. 6, https://esmed.org/MRA/mra/ article/view/1816/1866 (2018).

9. Saikaly SK, Khachemoune A. Honey and Wound Healing: An Update. Epub ahead of print .2017. DOI: $10.1007 / \mathrm{s} 40257-016$ 0247-8. 
10.Kurek-Górecka A, Górecki M, RzepeckaStojko A 2, et al. Bee Products in Dermatology and Skin Care, https://www. mdpi.com/1420-3049/25/3/556 (2020).

11.Zbuchea A. Up-to-date use of honey for burns treatment. 2014; XXVII: 22-30.

12. Oryan A, Alemzadeh E, Moshiri A. Biological properties and therapeutic activities of honey in wound healing: A narrative review and meta-analysis. J Tissue Viability. Epub ahead of print. 2016. DOI: 10.1016/j. jtv.2015.12.002.

13. Naseer S, Khan SA, Azim MK. Identification of cultivable bateria from natural honey of different botanical origin. 48, https://www. researchgate.net/publication/280038851_ Identification_of_cultivable_bacteria_from_ natural_honey_of_different_botanical_origin (2015).

14. Nweze JA, Olovo C V, Nweze EI, et al. Therapeutic Properties of Honey. 2019.

15. Molan P, Rhodes T. Honey: A Biologic Wound Dressing. 2015; 27: 141-151.

16. Morsi EAF, Mustafa FM, Tokhy A El. Vinegar Simple Method in Dressing of Pseudomonas Infected Wound. 3, https://www. researchgate.net/publication/332274982 Vinegar_Simple_Method in Dressing of Pseudomonas Infected_Wound (2016).

17. Pant PR, Towner E, Ellis M, et al. Epidemiology of Unintentional Child Injuries in the Makwanpur District of Nepal : A Household Survey. 2015; 15118-15128.

18. Mcdonald EM, Gielen AC. Injury Prevention : Falls. 2019; 1-7.
19. World Health Organization. Global status report on road safety. 2018; 1-424.

20. Patel A, Krebs E, Andrade L, et al. The epidemiology of road traffic injury hotspots in Kigali , Rwanda from police data. BMC Public Health. 2016; 1-10.

21. Wang D, Krebs E, Nickenig Vissoci JR, et al. Built Environment Analysis for Road Traffic Crash Hotspots in Kigali, Rwanda. Front Sustain Cities 2020; 2: 1-13.

22. Özkan A, Şentürk S, Tosun Z. Fasciotomy Procedures on Acute Compartment Syndromes of the Upper Extremity Related to Burns. 2015; 12: 326-331.

23. Stracciolini A, Hammerberg E. Acute compartment syndrome of the extremities.

24. Tulandi SM. The effect of storage temperature on the quality of honey. SANITAS J Teknol dan Seni Kesehat .2019; 08: 80-86.

25. Olaitan PB, Adeleke OE, Ola IO. Honey: A reservoir for microorganisms and an inhibitory agent for microbes. Afr Health Sci .2007; 7: 159-165.

26. Agrawal KS, Sarda AV, Shrotriya R, et al. Acetic acid dressings: Finding the Holy Grail for infected wound management. Epub ahead of print .2019. DOI: 10.4103/ijps.IJPS. 